\title{
ON SUPPORTING HYPERPLANES TO CONVEX BODIES*
}

\author{
ALESSIO FIGALLI ${ }^{\dagger}$, YOUNG-HEON KIM ${ }^{\ddagger}$, AND ROBERT J. MCCANN ${ }^{\S}$
}

\begin{abstract}
Given a convex set and an interior point close to the boundary, we prove the existence of a supporting hyperplane whose distance to the point is controlled, in a dimensionally quantified way, by the thickness of the convex set in the orthogonal direction. This result has important applications in the regularity theory for Monge-Ampère type equations arising in optimal transportation.
\end{abstract}

Key words. Convex bodies, supporting hyperplanes.

AMS subject classifications. 52A20, 52A40, 35J96.

1. Introduction. In this note we establish an estimate which quantifies the dimensional dependence of the claim that corresponding to any (interior) point near the boundary of a convex set, is a supporting hyperplane much closer than the thickness of the set in the orthogonal direction. The main interest of our estimate (see Theorem 1.1) is that it allows us [4] to extend — for the first time - a Hölder continuity result of Caffarelli $[3,2]$ concerning optimal transportation of bounded measurable densities from the quadratic cost function of Brenier [1], to the more general cost functions considered by Trudinger and Wang [7]. Caffarelli's regularity result has well-known connections to convex geometry (see [5]), and the importance of its extension to more general optimal transport problem is highlighted in [8].

Our theorem below is purely geometric, elementary to state, and non-trivial to prove; it may well be of independent interest. To emphasize this possibility, and the fact that it does not rely on any auxiliary concepts arising from the intended application [4], we establish it in this separate manuscript. By so doing, we hope to ensure its accessibility to convex geometers who may have no interest in optimal transportation, as well as to its primary target audience, which consists of researchers interested in the regularity of optimal mappings (or equivalently, of degenerate elliptic solutions to the associated Monge-Ampère type equations).

Let us start by recalling that a convex body $\tilde{S}$ in the $n$-dimensional Euclidean space $\boldsymbol{R}^{n}$ refers to a compact convex set with non-empty interior. A well-known result of Fritz John [6], often called John's Lemma, shows every convex body can be translated so that it contains an ellipsoid $E$ centered at the origin, whose dilation by factor $n$ contains the translated copy $S$ of $\tilde{S}$ :

$$
E \subset S \subset n E .
$$

*Received February 28, 2013; accepted for publication June 21, 2013. The authors are pleased to acknowledge the partial support of their research by United States' National Science Foundation grant DMS $0969962[\mathrm{AF}]$ and Natural Sciences and Engineering Research Council of Canada Grants 371642-09 [YHK] and 217006-08 [RJM]. Any opinions, findings and conclusions or recommendations expressed in this material are those of authors and reflect the views neither of the United States' National Science Foundation nor of the Natural Sciences and Engineering Research Council of Canada. $\mathrm{edu}$.

${ }^{\dagger}$ Department of Mathematics, University of Texas, Austin TX 78712, USA (figalli@math.utexas.

${ }_{\ddagger}^{\ddagger}$ Department of Mathematics, University of British Columbia, Vancouver BC Canada V6T 1Z2 (yhkim@math.ubc.ca).

$\S$ Department of Mathematics, University of Toronto, Toronto, Ontario Canada M5S 2E4 (mccann @math.toronto.edu). 
The constant $n$ is shown to be sharp by the standard simplex. After this translation, i.e. when (1.1) holds, we call $S$ well-centered. We restrict our discussion to only well-centered convex bodies, but this does not cost any generality.

For any point $\boldsymbol{y} \in S$ near the boundary of a well-centered convex body, we claim it is possible to find a direction in which the boundary of $S$ is much closer than the thickness of $S$ in the same direction. More precisely, we claim it is possible to find a line $L$ through the origin whose intersection with $S$ is large relative to the distance of $\boldsymbol{y}$ to a hyperplane outside of $S$ and orthogonal to $L$. Here orthogonal refers to the ambient Euclidean inner product, so that the two distances being compared are measured along line segments parallel to $L$. The following theorem quantifies the dependence of their ratio on the proximity of $\boldsymbol{y}$ to the boundary, as reflected in the degenerating factor $s^{1 / 2^{n-1}}$ in (1.2) below. When $\boldsymbol{y}$ approaches the boundary of $S$, the ratio of the two distances becomes more and more exaggerated, algebraically fast with respect to the separation of $\boldsymbol{y}$ from the boundary (but whose algebraic power decays exponentially fast in high dimensions). Here $s$ measures the separation of $\boldsymbol{y}$ from the boundary in the Minkowski gauge of $S$ - which of course is equivalent to any other norm on $\boldsymbol{R}^{n}$. In what follows however, 'dist', 'diam' (and orthogonality) always refer to distance and diameter with respect to the Euclidean norm. The application [4] requires only the special case $s_{0}=1 /(2 n)$.

TheOREM 1.1. Let $S \subset \boldsymbol{R}^{n}$ be a well-centered convex body, meaning (1.1) holds for some ellipsoid $E$ centered at the origin. Fix $0 \leq s \leq s_{0}<1$. For each $\boldsymbol{y} \in(1-s) \partial S$ there exists a hyperplane $P$ supporting $S$ such that

$$
\operatorname{dist}(\boldsymbol{y}, P) \leq c\left(n, s_{0}\right) s^{1 / 2^{n-1}} \operatorname{diam}\left(P^{\perp} \cap S\right) .
$$

Here $P^{\perp}$ denotes the (unique) line orthogonal to $P$ passing through the origin, and $c\left(n, s_{0}\right)$ is a constant depending only on $n$ and $s_{0}$, namely $c\left(n, s_{0}\right)=n^{3 / 2}(n-$ $\left.\frac{1}{2}\right)\left(\frac{1+\left(s_{0}\right)^{1 / 2^{n}}}{1-\left(s_{0}\right)^{1 / 2^{n}}}\right)^{n-1}$.

REMARK 1.2. For $n=1$, the constant $c\left(1, s_{0}\right)$ is sharp and (1.2) becomes an obvious equality since in this case $P$ is a point and $P^{\perp}$ is nothing but the line passing through the point and the origin; we have not investigated sharpness of $c\left(n, s_{0}\right)$ or of the power $2^{1-n}$ in higher dimensions.

The key point of the estimate (1.2) is that the ratio $\operatorname{dist}(\boldsymbol{y}, P) / \operatorname{diam}\left(P^{\perp} \cap S\right)$ goes to 0 as $s \rightarrow 0$ in a "uniform way", independent of the shape of $S$. Observe that if $s=0$ we can choose $P$ to support $S$ at $\boldsymbol{y} \in \partial S$, but for $s>0$ it is less obvious how to choose $P$ (and hence $L$ ). The difficulty for proving this estimate is on the arbitrariness of the convex body $S$. For example, if $S$ is the round ball, then the estimate (1.2) becomes trivial (indeed, one may even replace $c\left(n, s_{0}\right) s^{1 / 2^{n-1}}$ with $s$, and the supporting hyperplane $P$ shall be chosen to be orthogonal to the vector $\boldsymbol{y}$ ). For a general convex bodiy $S$, there are three natural ways to try to generalize such choice: (i) to choose $P$ orthogonal to $\boldsymbol{y}$, (ii) to choose $P$ supporting $S$ at the intersection of the half line $\overrightarrow{\mathbf{0 y}}$ with $\partial S$, or (iii) to choose $P$ closest to $\boldsymbol{y}$. However, in all these three cases it is not hard to find counterexamples (some family of degenerating thin convex bodies) showing such rather natural choices of hyperplanes do not work, namely, not yielding a uniform convergence to 0 of the ratio $\operatorname{dist}(\boldsymbol{y}, P) / \operatorname{diam}\left(P^{\perp} \cap S\right)$ as $s \rightarrow 0$. To prove Theorem 1.1 we find an algorithm which allows to choose appropriate $P$ by an inductive recursion, reducing the dimension of the task confronted at each step. 
One of the reasons why the estimate (1.2) is nontrivial is that the set of convex bodies is not compact. A common and powerful way to deal with such noncompactness is to use John's Lemma [6] (see (1.1)), to renormalize the convex bodies via affine maps so that the resulting shapes become roughly close to the round ball (with a uniformly bounded scale factor). This way, one can easily derive some estimates for quantities that are affine invariant. One such example is the classical Alexandrov estimates for the Monge-Ampère measure associated to sections of convex functions: see, for example [5]. However, in our case the inequality (1.2) involves orthogonality with respect to the fixed Euclidean norm, which is not affine invariant. Therefore, we cannot derive the estimate (1.2) by applying John's Lemma.

The remainder of this paper is devoted to the proof of Theorem 1.1, which is completely elementary though quite nontrivial.

2. Proof of Theorem 1.1. Let $S \subset \boldsymbol{R}^{n}$ be a well-centered convex body as in (1.1). Let the positive numbers $a^{1}, \cdots, a^{n} \in \boldsymbol{R}_{+}$denote the lengths of the principal semi-axes of the inner ellipsoid $E$ of Fritz John (1.1). One can regard these $a^{i}$ s as the coordinate components of the vector $\boldsymbol{a}=\left(a^{1}, \cdots, a^{n}\right)$. In the following, superscripts will be used to denote such coordinate components for vectors, and for all the other cases they will mean powers (we believe this should not create either confusion or ambiguity).

Use these principal axes to choose coordinates, with the origin $\mathbf{0}$ at the center of $E$. We still have the freedom to choose the order in which these axes are enumerated, which we shall exploit especially at (2.9). In these coordinates $E$ is represented as

$$
E=\left\{\boldsymbol{x}=\left(x^{1}, \cdots, x^{n}\right) \in \boldsymbol{R}^{n} \mid \sum_{i=1}^{n}\left(\frac{x^{i}}{a^{i}}\right)^{2} \leq 1\right\} .
$$

The rectangle

$$
R_{n}=\left\{\boldsymbol{x}=\left(x^{1}, \cdots, x^{n}\right) \in \boldsymbol{R}^{n}|| x^{i} \mid \leq n a^{i}, i=1, \cdots, n\right\}
$$

circumscribed around the outer ellipse $n E$ will also play a crucial role. Observe from our construction of $R_{n}$ and (1.1), $\frac{1}{n^{3 / 2}} R_{n} \subset S \subset R_{n}$. In particular, $S$ is comparable (in size and shape) to $R_{n}$.

2.1. Initial step in the recursive algorithm. Fix $s_{0} \in(0,1)$, and given $\boldsymbol{y} \in(1-s) \partial S, 0 \leq s \leq s_{0}$, let $\boldsymbol{p}_{n}$ be the intersection of the half line $\overrightarrow{\mathbf{0}} \boldsymbol{y}$ with $\partial S$. We pick a tangent hyperplane $P_{n}$ (which may not be unique) to $S$ at $\boldsymbol{p}_{n}$. Using similar triangles, we deduce that

$$
\frac{\operatorname{dist}\left(\boldsymbol{y}, P_{n}\right)}{\operatorname{dist}\left(\mathbf{0}, P_{n}\right)}=s
$$

Two alternatives. We consider the following two exclusive cases:

Favorable case: Suppose we are lucky enough that

$$
\frac{\operatorname{diam}\left(P_{n}^{\perp} \cap R_{n}\right)}{2 \operatorname{dist}\left(\mathbf{0}, P_{n}\right)} \geq s^{1 / 2}
$$


Then, the choice $P=P_{n}$ leads to the desired result (1.2) since $\operatorname{diam}\left(P_{n}^{\perp} \cap R_{n}\right) \leq$ $n^{3 / 2} \operatorname{diam}\left(P_{n}^{\perp} \cap S\right)$, thus

$$
\begin{aligned}
\frac{\operatorname{dist}\left(\boldsymbol{y}, P_{n}\right)}{\operatorname{diam}\left(P_{n}^{\perp} \cap S\right)} & \leq n^{3 / 2} \frac{\operatorname{dist}\left(\boldsymbol{y}, P_{n}\right)}{\operatorname{dist}\left(\mathbf{0}, P_{n}\right)} \frac{\operatorname{dist}\left(\mathbf{0}, P_{n}\right)}{\operatorname{diam}\left(P_{n}^{\perp} \cap R_{n}\right)} \\
& \leq n^{3 / 2} \frac{s}{2 s^{1 / 2}} \\
& =\frac{n^{3 / 2}}{2} s^{1 / 2} .
\end{aligned}
$$

Unfavorable case: If the convex body $S$ is very thin, or equivalently if the outer rectangle $R_{n}$ is very thin, then the ratio $\operatorname{diam}\left(P_{n}^{\perp} \cap R_{n}\right) / 2 \operatorname{dist}\left(\mathbf{0}, P_{n}\right)$ can be much smaller than $s^{1 / 2}$, in which case (2.1) fails. For such situations, we now describe a recursive algorithm which shows that whenever (2.1) is violated, after at most $(n-1)$ steps it is possible to find an alternative hyperplane $P$ (generally different from $P_{n}$ ) which fulfils the desired conclusion (1.2).

2.2. Notation in the recursive algorithm. The basic idea of the following recursive algorithm is to repeat the previous two alternatives in the inductive steps, with decreasing dimension. Since this is a finite dimensional situation, such algorithm should terminate, and we show it does so yielding the desired result (1.2). One of the key points of the argument is to choose the right geometric configuration. This requires in particular some careful choice of the terms $\gamma_{i}(s), \delta_{i}(s)$, and $c_{0}$, as we fix the notation below.

Let $i, k \in\{1, \ldots, n\}$ and $s \in\left[0, s_{0}\right]$. We define

$$
\begin{aligned}
& \gamma_{i}(s):=s^{1 / 2^{i}} \\
& \delta_{i}(s):=(2 i-1) s^{1 / 2^{i-1}} .
\end{aligned}
$$

These satisfy the following key relations:

$$
\begin{aligned}
\delta_{i+1}(s) & \geq \delta_{i}(s)+2 \gamma_{i}(s) ; \\
\frac{\delta_{i}(s)}{\gamma_{i}(s)} & =(2 i-1) \gamma_{i}(s) .
\end{aligned}
$$
$\boldsymbol{R}^{k}$ by

We use coordinates $\left(x^{1}, \cdots, x^{k+1}\right)$ on $\boldsymbol{R}^{k+1}$. Define the projections $\pi_{k}: \boldsymbol{R}^{k+1} \rightarrow$

$$
\pi_{k}\left(x^{1}, \cdots, x^{k}, x^{k+1}\right)=\left(x^{1}, \cdots, x^{k}\right) .
$$

Observe that each $\pi_{k}$ is determined by the choice of the coordinate axis for $x^{k+1}$ for $\boldsymbol{R}^{k+1}$, and such choice will be made individually at each step of the recursive algorithm. This is an important point to remember throughout the proof. For $k<n$, define the rectangles $R_{k}$ in $\boldsymbol{R}^{k}$ inductively as dilated projections of $R_{n}$ :

$$
R_{k}=c_{0} \pi_{k}\left(R_{k+1}\right) .
$$

Here, the dilation factor $c_{0}$ (with respect to the origin $\mathbf{0}$ ) is given by

$$
\begin{aligned}
c_{0} & :=\max _{0 \leq s \leq s_{0} ; 1 \leq i \leq n}\left[1+2 \frac{\gamma_{i}(s)}{1-\gamma_{i}(s)}\right] \\
& =\frac{1+\left(s_{0}\right)^{1 / 2^{n}}}{1-\left(s_{0}\right)^{1 / 2^{n}}},
\end{aligned}
$$


where the monotone dependence of $\gamma_{i}(s)$ on both $s \leq s_{0}$ and $i \leq n$ has been used. These rectangles can also be written as

$$
R_{k}=\left\{\boldsymbol{x}=\left(x^{1}, \cdots, x^{k}\right) \in \boldsymbol{R}^{k}|| x^{i} \mid \leq c_{0}^{n-k} n a^{i}, \quad 1 \leq i \leq k\right\} .
$$

Let $Q_{k}^{ \pm}$(and $Q_{k}^{0}$ ) be the parallel hyperplanes in $\boldsymbol{R}^{k}$ which form the boundary of (and bisect) $R_{k}$ orthogonally to the $x^{k}$-axis:

$$
\begin{aligned}
Q_{k}^{ \pm} & =\left\{\boldsymbol{x}=\left(x^{1}, \cdots, x^{k}\right) \in \boldsymbol{R}^{k} \mid x^{k}= \pm c_{0}^{n-k} n a^{k}\right\} \\
Q_{k}^{0} & =\left\{\boldsymbol{x}=\left(x^{1}, \cdots, x^{k}\right) \in \boldsymbol{R}^{k} \mid x^{k}=0\right\} .
\end{aligned}
$$

Set $\boldsymbol{y}_{n}=\boldsymbol{y}=\left(y^{1}, \ldots, y^{n}\right)$ and define its projections recursively

$$
\boldsymbol{y}_{k}:=\pi_{k} \circ \pi_{k+1} \circ \cdots \circ \pi_{n-1}(\boldsymbol{y})=\left(y^{1}, \ldots, y^{k}\right) .
$$

Since $c_{0} \geq 1$ it is clear that $\boldsymbol{y}_{k} \in R_{k}$.

In the following we will define some hyperplanes $P_{k} \subset \boldsymbol{R}^{k}$ inductively. For such a hyperplane $P_{k} \subset \boldsymbol{R}^{k}$ use $\tilde{P}_{k}$ to denote the extension of $P_{k}$ to the hyperplane in $\boldsymbol{R}^{n}$ parallel to the $x^{k+1}$ - through $x^{n}$-axes, i.e., which satisfies

$$
\pi_{k} \circ \cdots \circ \pi_{n-1}\left(\tilde{P}_{k}\right)=P_{k} .
$$

2.3. The structure of the recursive algorithm. Recall the point $\boldsymbol{p}_{n}=\overrightarrow{\mathbf{0 y}} \cap$ $\partial S$ and the supporting hyperplane $P_{n}$, with $\boldsymbol{p}_{n} \in P_{n}$, which are given in the initial step of the recursive algorithm (Section 2.1).

Let us describe the recursion in which we use $\left(\boldsymbol{p}_{k}, P_{k}\right)$ to define $\left(\boldsymbol{p}_{k-1}, P_{k-1}\right)$. We first list three conditions that are required at each step of the recursive algorithm. For the $i^{t h}$ step (here $i=n-k+1$ ) assume that

$$
\begin{array}{ll}
\mathbf{A}_{k}: & \boldsymbol{p}_{k} \in P_{k} \cap R_{k}, \quad \boldsymbol{y}_{k} \in\left[\mathbf{0}, \boldsymbol{p}_{k}\right] ; \\
\mathbf{B}_{k}: & \frac{\operatorname{dist}\left(\boldsymbol{y}_{k}, P_{k}\right)}{\operatorname{dist}\left(\mathbf{0}, P_{k}\right)} \leq \delta_{n-k+1}(s) ; \\
\mathbf{C}_{k}: & \tilde{P}_{k} \text { does not intersect the interior of } S .
\end{array}
$$

Notice that for $k=n$, either the favorable situation (2.1) holds, in which case there is nothing further to prove (and so no need to proceed to the next step), or else $\mathbf{A}_{n}, \mathbf{B}_{n}, \mathbf{C}_{n}$ are satisfied by our initial choice of $\boldsymbol{p}_{n}$ and $P_{n}$.

Starting from $k=n$, we shall decrease $k$ one step at a time until the algorithm terminates. Whether or not the recursion terminates at a given value of $k$ is determined by the following dichotomy:

$$
\begin{array}{ll}
\text { Case I (favorable case): } & \frac{\operatorname{diam}\left(P_{k}^{\perp} \cap R_{k}\right)}{2 \operatorname{dist}\left(\mathbf{0}, P_{k}\right)} \geq \gamma_{n-k+1}(s) ; \\
\text { Case II (unfavorable case): } & \frac{\operatorname{diam}\left(P_{k}^{\perp} \cap R_{k}\right)}{2 \operatorname{dist}\left(\mathbf{0}, P_{k}\right)}<\gamma_{n-k+1}(s) .
\end{array}
$$

If Case I holds for some value of $k$ we shall discover we are in a favorable situation - analogous to (2.1) - which allows us to terminate the recursion and obtain the desired result (1.2). On the other hand, if Case II holds for the given value of $k$, 
we shall see we can use $\left(\boldsymbol{p}_{k}, P_{k}\right)$ satisfying the inductive hypotheses $\mathbf{A}_{k}, \mathbf{B}_{k}$ and $\mathbf{C}_{k}$, and the condition (2.7), to construct $\left(\boldsymbol{p}_{k-1}, P_{k-1}\right)$ satisfying $\mathbf{A}_{k-1}, \mathbf{B}_{k-1}$ and $\mathbf{C}_{k-1}$. We then decrease $k$ and proceed to the next step of the recursion. In the worst case the recursion continues until $k=1$, and we find $\left(p_{1}, P_{1}\right)$ satisfying $\mathbf{A}_{1}, \mathbf{B}_{1}$ and $\mathbf{C}_{1}$. In this case we show in the last section below that the desired result (1.2) can again be obtained, to complete the proof of the theorem.

2.4. Case I, (2.6) holds for some $k \geq 2$ : the recursion terminates with the desired result. As soon as we reach some $k \geq 2$ for which the condition (2.6) holds, we stop the recursion. We now show in this case the desired result (1.2) follows. Here, the assumptions $\mathbf{B}_{k}$ and $\mathbf{C}_{k}$ are crucial. Since, by the construction of $\boldsymbol{y}_{k}$ and $\tilde{P}_{k}, \operatorname{dist}\left(\boldsymbol{y}, \tilde{P}_{k}\right)=\operatorname{dist}\left(\boldsymbol{y}_{k}, P_{k}\right)$ and $\operatorname{dist}\left(\mathbf{0}, \tilde{P}_{k}\right)=\operatorname{dist}\left(\mathbf{0}, P_{k}\right)$, recalling (2.5) we get

$$
\begin{aligned}
\frac{\operatorname{dist}\left(\boldsymbol{y}, \tilde{P}_{k}\right)}{c_{0}^{n-k} \operatorname{diam}\left(\tilde{P}_{k}^{\perp} \cap R_{n}\right)} & =\frac{\operatorname{dist}\left(\boldsymbol{y}_{k}, P_{k}\right)}{\operatorname{dist}\left(\mathbf{0}, P_{k}\right)} \frac{\operatorname{dist}\left(\mathbf{0}, P_{k}\right)}{\operatorname{diam}\left(P_{k}^{\perp} \cap R_{k}\right)} \\
& \leq \frac{\delta_{n-k+1}(s)}{2 \gamma_{n-k+1}(s)} \quad\left(\text { by }(2.6) \text { and } \mathbf{B}_{k}\right) .
\end{aligned}
$$

Let $\tilde{H}_{k}$ be the half-space containing $\mathbf{0}$, with $\partial \tilde{H}_{k}=\tilde{P}_{k}$. Notice that $S \subset \tilde{H}_{k}$ by assumption $\mathbf{C}_{k}$. Thus, translating $\tilde{P}_{k}$ toward $S$, one can find a hyperplane $P$ supporting $S$, which is parallel to $\tilde{P}_{k}$. Since $\operatorname{dist}(\boldsymbol{y}, P) \leq \operatorname{dist}\left(\boldsymbol{y}, \tilde{P}_{k}\right)$ and $\operatorname{diam}\left(P^{\perp} \cap S\right)=$ $\operatorname{diam}\left(\tilde{P}_{k}^{\perp} \cap S\right) \geq n^{-3 / 2} \operatorname{diam}\left(\tilde{P}_{k}^{\perp} \cap R_{n}\right)$, from (2.2) and (2.8) we have

$$
\begin{aligned}
\frac{\operatorname{dist}(\boldsymbol{y}, P)}{\operatorname{diam}\left(P^{\perp} \cap S\right)} & \leq n^{3 / 2} c_{0}^{n-k} \frac{\delta_{n-k+1}(s)}{2 \gamma_{n-k+1}(s)} \\
& =n^{3 / 2} c_{0}^{n-k}\left(n-k+\frac{1}{2}\right) s^{1 / 2^{n-k+1}} \\
& \leq n^{3 / 2} c_{0}^{n-1}\left(n-\frac{3}{2}\right) s^{1 / 2^{n-1}}
\end{aligned}
$$

(recall that $k \geq 2$ ), which gives the desired result (1.2).

2.5. Case II, (2.7) holds for $2 \leq k \leq n$ : the recursion continues. A remark before we proceed: in the following argument, we assume that any claimed intersections between affine subspaces such as lines and (hyper-)planes actually exist and have the expected (i.e. generic) dimension. This costs no generality for two reasons:

- To avoid parallelism we can perturb if necessary (i.e. rotate and/or translate slightly) the affine subspaces.

- We will obtain estimates which are not sensitive to small perturbations, so the estimates also hold without the perturbation. Moreover, the obtained bounds then imply that the claimed intersections do indeed exist.

Similarly, we can also avoid, if necessary, the cases where some lengths and/or distances degenerate to zero.

Some preliminaries and the construction of $P_{k-1}$. To define $P_{k-1}$ and $\boldsymbol{p}_{k-1}$ we set-up preliminaries. Let us first consider the point $\boldsymbol{r}_{k}=\left(r_{k}^{1}, \cdots, r_{k}^{k}\right) \in \boldsymbol{R}^{k}$ defined as the closest point on $P_{k}$ to the origin, so that $\operatorname{dist}\left(\mathbf{0}, \boldsymbol{r}_{k}\right)=\operatorname{dist}\left(\mathbf{0}, P_{k}\right)$. Note $\boldsymbol{r}_{k}$ is outside the rectangle $R_{k}$, since otherwise,

$$
\frac{\operatorname{diam}\left(P_{k}^{\perp} \cap R_{k}\right)}{2 \operatorname{dist}\left(\mathbf{0}, P_{k}\right)} \geq 1
$$




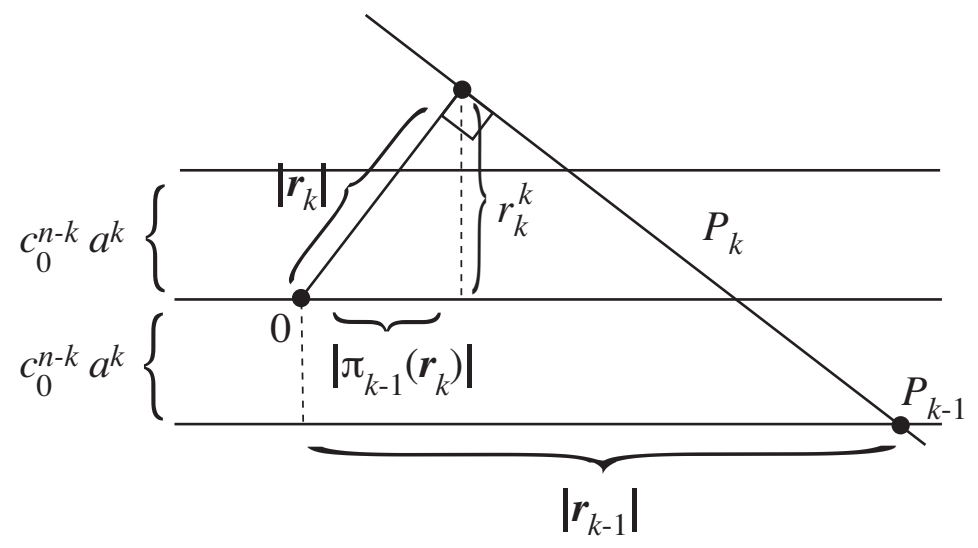

FIG. 1. By a simple argument based on similar triangles, one can easily compute $\left|\pi_{k-1}\left(\boldsymbol{r}_{k}\right)\right|$ in terms of $\left|\boldsymbol{r}_{k}\right|, c_{0}^{n-k} a^{k}$, and $r_{k}^{k}$.

contradicting assumption $(2.7)$. Let $\boldsymbol{r}_{k}^{+}=\left(\left(\boldsymbol{r}_{k}^{+}\right)^{1}, \cdots,\left(\boldsymbol{r}_{k}^{+}\right)^{k}\right)=\left[0, \boldsymbol{r}_{k}\right] \cap \partial R_{k}$ denote the intersection point of the ray through $\boldsymbol{r}_{k}$ with whichever of the $2 k$ faces of this rectangle it intersects. Without loss of generality, suppose the axes are enumerated so that the intersection occurs on the face of $R_{k}$ contained in $Q_{k}^{+}$. (Observe that this choice of coordinates affects the definition of $\pi_{k-1}$.) Then,

$$
\left(\boldsymbol{r}_{k}^{+}\right)^{k}=c_{0}^{n-k} a^{k}>0
$$

holds. Because $\operatorname{dist}\left(\mathbf{0}, \boldsymbol{r}_{k}^{+}\right)=\frac{1}{2} \operatorname{diam}\left(P_{k}^{\perp} \cap R_{k}\right)$ and $\operatorname{dist}\left(\mathbf{0}, \boldsymbol{r}_{k}\right)=\operatorname{dist}\left(\mathbf{0}, P_{k}\right)$, we have

$$
\frac{\operatorname{dist}\left(\mathbf{0}, \boldsymbol{r}_{k}^{+}\right)}{\operatorname{dist}\left(\mathbf{0}, \boldsymbol{r}_{k}\right)}=\frac{\operatorname{diam}\left(P_{k}^{\perp} \cap R_{k}\right)}{2 \operatorname{dist}\left(\mathbf{0}, P_{k}\right)}
$$

Now, define the hyperplane $P_{k-1}$ in $\boldsymbol{R}^{k-1}$ by

$$
P_{k-1}:=\pi_{k-1}\left(P_{k} \cap Q_{k}^{-}\right)
$$

Verification of $\mathbf{C}_{k-1}$. Before proceeding further, let us verify that $\mathbf{C}_{k-1}$ follows from $\mathbf{C}_{k}$ as a consequence.

Since $r_{k}^{k}=\sqrt{\left|\boldsymbol{r}_{k}\right|^{2}-\left|\pi_{k-1}\left(\boldsymbol{r}_{k}\right)\right|^{2}}$, by a simple geometric argument (see Figure 1) the construction above yields $\boldsymbol{r}_{k-1}=\lambda \pi_{k-1}\left(\boldsymbol{r}_{k}\right)$ (recall that $\boldsymbol{r}_{k-1}$ is defined as the closest point on $P_{k-1}$ to the origin), with $\lambda=\left(\left|\boldsymbol{r}_{k}\right|^{2}+c_{0}^{n-k} a^{k} r_{k}^{k}\right) /\left|\pi_{k-1}\left(\boldsymbol{r}_{k}\right)\right|^{2}>1$.

For each $\boldsymbol{x}=\left(x^{1}, \ldots, x^{n}\right)=: \boldsymbol{x}_{n}$ from the interior of $S$, let $\boldsymbol{x}_{k}:=\pi_{k} \circ \pi_{k+1} \ldots \circ$ $\pi_{n-1}(\boldsymbol{x})=\left(x^{1}, \ldots, x^{k}\right)$. To verify $\mathbf{C}_{k-1}$ we need to show that $\boldsymbol{x}_{k-1} \cdot \boldsymbol{r}_{k-1} \leq\left|\boldsymbol{r}_{k-1}\right|^{2}$. Without loss of generality assume that $\boldsymbol{x}_{k-1} \cdot \boldsymbol{r}_{k-1}>0$. Hence, since $\lambda>1$,

$$
\begin{aligned}
\boldsymbol{x}_{k-1} \cdot \boldsymbol{r}_{k-1} & <\lambda\left(\boldsymbol{x}_{k} \cdot \boldsymbol{r}_{k}+a^{k} r_{k}^{k}\right) \\
& <\lambda\left(\left|\boldsymbol{r}_{k}\right|^{2}+a^{k} r_{k}^{k}\right) \\
& \leq\left|\boldsymbol{r}_{k-1}\right|^{2},
\end{aligned}
$$

where the first inequality follows from $x^{k}>-a^{k}$, the second from $\mathbf{C}_{k}$, and the third from $c_{0} \geq 1$ and the definition of $\lambda$. This yields $\mathbf{C}_{k-1}$ as desired. 


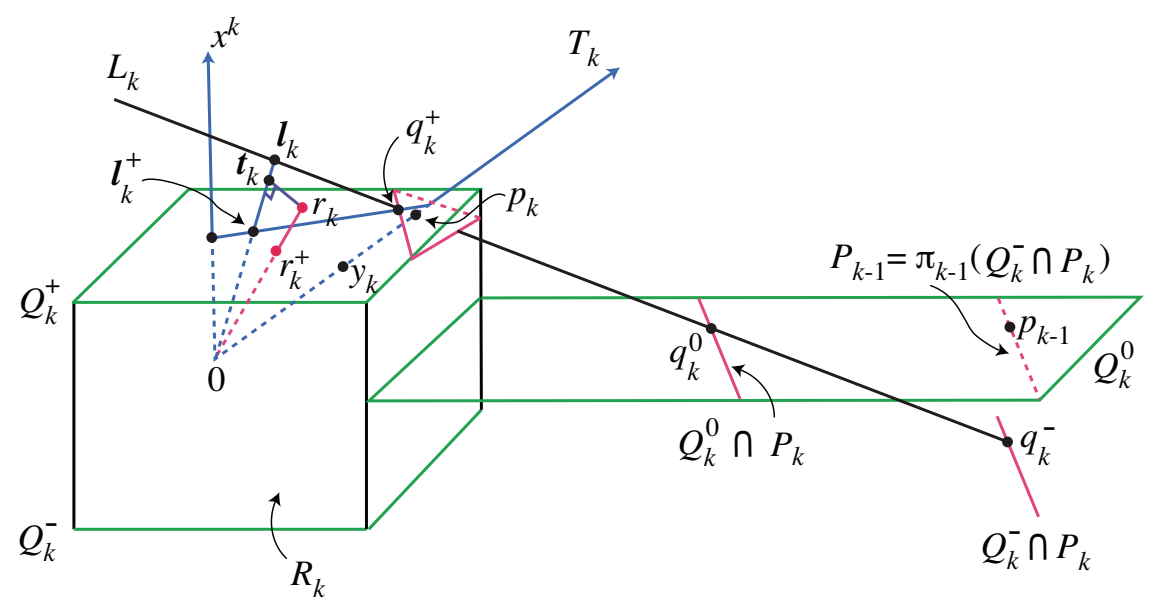

FIG. 2. The geometric argument behind the construction of $\boldsymbol{p}_{k-1}$. Observe that, for $s$ small, this figure (as well as the other figures) is not very "realistic", as $R_{k}$ should be very thin in the "horizontal" directions and $P_{k-1}$ very close to $R_{k}$. However, even if the proportions are not respected, this figure should help the reader to follow the argument described below. Note that one can construct convex sets for which $\boldsymbol{y}_{k}$ and $\boldsymbol{p}_{k}$ may lie below $Q_{k}^{0}$. However, although the picture is just indicative, our proof is purely analytic and works independently of the position of $\boldsymbol{y}_{k}$ and $\boldsymbol{p}_{k}$ with respect to $Q_{k}^{0}$.

Construction of $\boldsymbol{p}_{k-1}$. We will now find $\boldsymbol{p}_{k-1} \in \boldsymbol{R}^{k-1}$ so that $\mathbf{A}_{k-1}$ and $\mathbf{B}_{k-1}$ are satisfied. To define $\boldsymbol{p}_{k-1}$, consider the two-dimensional plane $T_{k} \subset \boldsymbol{R}^{k}$, generated by the $x^{k}$-axis and the half-line $\overrightarrow{\mathbf{0} \boldsymbol{p}_{k}}$ (which is the same as $\overrightarrow{\mathbf{0} \boldsymbol{y}_{k}}$ ). (We perturb $\boldsymbol{p}_{k}$ slightly if necessary to ensure it does not lie on the $x^{k}$-axis.) Since $\operatorname{dim} T_{k}+\operatorname{dim} P_{k}=k+1$, the affine intersection $L_{k}:=T_{k} \cap P_{k} \subset \boldsymbol{R}^{k}$ contains at least a line; it contains at most a line since $\mathbf{0} \in T_{k} \backslash P_{k}$. Notice that the line $L_{k}$ passes through the point $\boldsymbol{p}_{k}$ and the hyperplane $Q_{k}^{-}$, as in Figure 2 .

We define

$$
\boldsymbol{p}_{k-1}:=\pi_{k-1}\left(L_{k} \cap Q_{k}^{-}\right)
$$

Notice that

$$
\boldsymbol{p}_{k-1} \in P_{k-1}, \quad \boldsymbol{y}_{k-1} \in\left[\mathbf{0}, \boldsymbol{p}_{k-1}\right] .
$$

In particular, to verify $\mathbf{A}_{k-1}$ we only need to check $\boldsymbol{p}_{k-1} \in R_{k-1}$.

Preparation before verifying $\mathbf{A}_{k-1}$ and $\mathbf{B}_{k-1}$. To verify $\mathbf{A}_{k-1}$ and $\mathbf{B}_{k-1}$ we first find a few relevant points on the plane $T_{k}$. What we are going to describe is summarized in Figure 2.

The line $L_{k}$ intersects with the three parallel hyperplanes $Q_{k}^{0}, Q_{k}^{ \pm} \subset \boldsymbol{R}^{k}$. Denote the three intersection points by

$$
\begin{aligned}
\boldsymbol{q}_{k}^{+} & :=L_{k} \cap Q_{k}^{+} ; \\
\boldsymbol{q}_{k}^{0} & :=L_{k} \cap Q_{k}^{0} ; \\
\boldsymbol{q}_{k}^{-} & :=L_{k} \cap Q_{k}^{-} .
\end{aligned}
$$




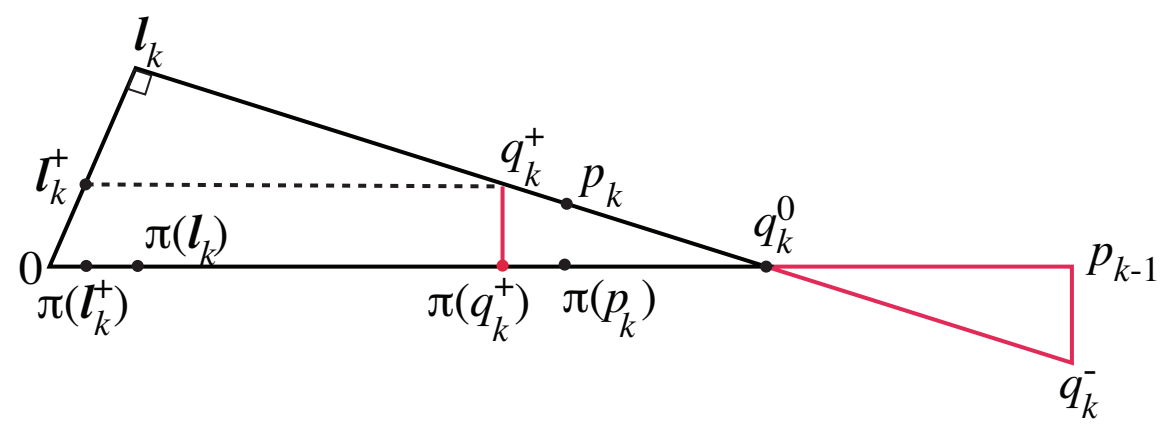

Fig. 3. Some useful similitudes. Note that, analogously to what observed in the comment to Figure 2, it maybe also possible that $\boldsymbol{p}_{k}\left(\right.$ resp, $\pi_{k-1}\left(\boldsymbol{p}_{k}\right)$ ) be located between $\boldsymbol{q}_{k}^{0}$ and $\boldsymbol{q}_{k}^{-}$(resp, $\left.\boldsymbol{p}_{k-1}\right)$.

Notice that $\boldsymbol{p}_{k} \in\left[\boldsymbol{q}_{k}^{+}, \boldsymbol{q}_{k}^{-}\right]$and $\pi_{k-1}\left(\boldsymbol{q}_{k}^{-}\right)=\boldsymbol{p}_{k-1}$.

Let $\boldsymbol{l}_{k}$ denote the closest point on $L_{k}$ to the origin $\mathbf{0}$, and let $\boldsymbol{t}_{k} \in T_{k}$ be the orthogonal projection of $\boldsymbol{r}_{k}$ on the plane $T_{k}$. Notice that, since $\boldsymbol{r}_{k}$ is the closest point in $P_{k}$ to $\mathbf{0}$ and $L_{k} \subset P_{k}$, the orthogonal projection of the ray $\overrightarrow{\mathbf{0} \boldsymbol{r}_{k}}$ to $T_{k}$ is the ray $\overrightarrow{\mathbf{0 l}_{k}}$ and $\left|\boldsymbol{r}_{k}\right| \leq\left|\boldsymbol{l}_{k}\right|$ (to see this, one may consider the plane passing through $\mathbf{0}, \boldsymbol{r}_{k}$, and $\boldsymbol{l}_{k}$, and observe that it cuts $L_{k}$ orthogonally). In particular, the point $\boldsymbol{t}_{k}$ belongs to the line segment $\left[\mathbf{0}, \boldsymbol{l}_{k}\right]$. Moreover, since $T_{k}$ contains the $x^{k}$-axis, $t_{k}^{k}=r_{k}^{k}$. Hence, by our assumption $(2.9), \boldsymbol{t}_{k}$ belongs to the region over $Q_{k}^{+}$, namely $t_{k}^{k}>c_{0}^{n-k} a^{k}$, as (therefore) does $\boldsymbol{l}_{k}$. Consider the point

$$
\boldsymbol{l}_{k}^{+}:=\left[\mathbf{0}, \boldsymbol{l}_{k}\right] \cap Q_{k}^{+}
$$

with $\left(\boldsymbol{l}_{k}^{+}\right)^{k}=\left(\boldsymbol{r}_{k}^{+}\right)^{k}=c_{0}^{n-k} a^{k}$. Since (as we observed above) $t_{k}^{k}=r_{k}^{k}$, the triangles $\triangle\left(\boldsymbol{t}_{k}, \mathbf{0}, \boldsymbol{r}_{k}\right)$ and $\triangle\left(\boldsymbol{l}_{k}^{+}, \mathbf{0}, \boldsymbol{r}_{k}^{+}\right)$are similar. Thus

$$
\frac{\operatorname{dist}\left(\mathbf{0}, \boldsymbol{l}_{k}^{+}\right)}{\operatorname{dist}\left(\mathbf{0}, \boldsymbol{l}_{k}\right)} \leq \frac{\operatorname{dist}\left(\mathbf{0}, \boldsymbol{l}_{k}^{+}\right)}{\operatorname{dist}\left(\mathbf{0}, \boldsymbol{t}_{k}\right)}=\frac{\operatorname{dist}\left(\mathbf{0}, \boldsymbol{r}_{k}^{+}\right)}{\operatorname{dist}\left(\mathbf{0}, \boldsymbol{r}_{k}\right)} \leq \gamma_{n-k+1}(s) .
$$

Here, the last inequality follows from (2.10) and (2.7).

Now the triangle $\triangle\left(\mathbf{0}, \boldsymbol{l}_{k}, \boldsymbol{q}_{k}^{0}\right)$ formed by the three points $\mathbf{0}, \boldsymbol{l}_{k}$ and $\boldsymbol{q}_{k}^{0}$ has a right angle at $\boldsymbol{l}_{k}$. This entire triangle projects to a line segment in $\boldsymbol{R}^{k-1}$, with the projection $\pi_{k-1}$ preserving the order of points and ratios of distances along the edges of $\triangle\left(\mathbf{0}, \boldsymbol{l}_{k}, \boldsymbol{q}_{k}^{0}\right)$ as in Figure 3 - a fact we shall continue to use subsequently.

Similarity to $\triangle\left(\boldsymbol{l}_{k}^{+}, \boldsymbol{l}_{k}, \boldsymbol{q}_{k}^{+}\right)$combines with (2.14) to yield

$$
\frac{\operatorname{dist}\left(\pi_{k-1}\left(\boldsymbol{q}_{k}^{0}\right), \pi_{k-1}\left(\boldsymbol{q}_{k}^{+}\right)\right)}{\operatorname{dist}\left(\pi_{k-1}\left(\boldsymbol{q}_{k}^{0}\right), \pi_{k-1}\left(\boldsymbol{l}_{k}\right)\right)}=\frac{\operatorname{dist}\left(\boldsymbol{q}_{k}^{0}, \boldsymbol{q}_{k}^{+}\right)}{\operatorname{dist}\left(\boldsymbol{q}_{k}^{0}, \boldsymbol{l}_{k}\right)}=\frac{\operatorname{dist}\left(\mathbf{0}, \boldsymbol{l}_{k}^{+}\right)}{\operatorname{dist}\left(\mathbf{0}, \boldsymbol{l}_{k}\right)} \leq \gamma_{n-k+1}(s) .
$$

Now we are ready to verify $\mathbf{A}_{k-1}$ and $\mathbf{B}_{k-1}$.

\section{Verification of $\mathbf{A}_{k-1}$.}

Claim 2.1. Definitions (2.11)-(2.12) imply $\mathbf{A}_{k-1}$.

Proof. By (2.13) it is enough to show that $\boldsymbol{p}_{k-1} \in R_{k-1}$. This should be clear from the geometric construction. Here the factor $c_{0}$ in the definition $R_{k-1}=c_{0} \pi_{k-1}\left(R_{k}\right)$ plays a crucial role. To give details, first note that $\mathbf{A}_{k}$ implies

$$
\pi_{k-1}\left(\boldsymbol{p}_{k}\right) \in \pi_{k-1}\left(R_{k}\right) \text {. }
$$


From $\boldsymbol{p}_{k} \in\left[\boldsymbol{q}_{k}^{+}, \boldsymbol{q}_{k}^{-}\right]$and $\boldsymbol{p}_{k-1}=\pi_{k-1}\left(\boldsymbol{q}_{k}^{-}\right)$, we have

$$
\begin{aligned}
\operatorname{dist}\left(\mathbf{0}, \boldsymbol{p}_{k-1}\right) & =\operatorname{dist}\left(\mathbf{0}, \pi_{k-1}\left(\boldsymbol{p}_{k}\right)\right)+\operatorname{dist}\left(\pi_{k-1}\left(\boldsymbol{p}_{k}\right), \boldsymbol{p}_{k-1}\right) \\
& \leq \operatorname{dist}\left(\mathbf{0}, \pi_{k-1}\left(\boldsymbol{p}_{k}\right)\right)+\operatorname{dist}\left(\pi_{k-1}\left(\boldsymbol{q}_{k}^{+}\right), \boldsymbol{p}_{k-1}\right) \\
& =\operatorname{dist}\left(\mathbf{0}, \pi_{k-1}\left(\boldsymbol{p}_{k}\right)\right)+2 \operatorname{dist}\left(\pi_{k-1}\left(\boldsymbol{q}_{k}^{+}\right), \pi_{k-1}\left(\boldsymbol{q}_{k}^{0}\right)\right) .
\end{aligned}
$$

Here, to bound the last line, observe that from (2.15),

$$
\operatorname{dist}\left(\pi_{k-1}\left(\boldsymbol{q}_{k}^{+}\right), \pi_{k-1}\left(\boldsymbol{q}_{k}^{0}\right)\right) \leq \frac{\gamma_{n-k+1}(s)}{1-\gamma_{n-k+1}(s)} \operatorname{dist}\left(\pi_{k-1}\left(\boldsymbol{l}_{k}\right), \pi_{k-1}\left(\boldsymbol{q}_{k}^{+}\right)\right) .
$$

From the geometry of the right triangle $\triangle\left(\mathbf{0}, \boldsymbol{l}_{k}, \boldsymbol{q}_{k}^{0}\right) \subset T_{k}$,

$$
\operatorname{dist}\left(\pi_{k-1}\left(\boldsymbol{l}_{k}\right), \pi_{k-1}\left(\boldsymbol{q}_{k}^{+}\right)\right) \leq \operatorname{dist}\left(\mathbf{0}, \pi_{k-1}\left(\boldsymbol{p}_{k}\right)\right) .
$$

Recalling that $\boldsymbol{p}_{k-1}$ is parallel to $\pi_{k-1}\left(\boldsymbol{p}_{k}\right)$, combining the preceding four displayed statements with (2.3) yields $\boldsymbol{p}_{k-1} \in c_{0} \pi_{k-1}\left(R_{k}\right)=R_{k-1}$ as desired. This completes the proof of Claim 2.1.

\section{Verification of $\mathbf{B}_{k-1}$.}

Claim 2.2. Definitions (2.11)-(2.12) imply $\mathbf{B}_{k-1}$.

Proof. Since $\boldsymbol{y}_{k-1} \in\left[\mathbf{0}, \boldsymbol{p}_{k-1}\right]$, from similarity

$$
\frac{\operatorname{dist}\left(\boldsymbol{y}_{k-1}, P_{k-1}\right)}{\operatorname{dist}\left(\mathbf{0}, P_{k-1}\right)}=\frac{\operatorname{dist}\left(\boldsymbol{y}_{k-1}, \boldsymbol{p}_{k-1}\right)}{\operatorname{dist}\left(\mathbf{0}, \boldsymbol{p}_{k-1}\right)} \text {. }
$$

To bound the latter:

$$
\begin{aligned}
& \operatorname{dist}\left(\boldsymbol{y}_{k-1}, \boldsymbol{p}_{k-1}\right) \\
& =\operatorname{dist}\left(\pi_{k-1}\left(\boldsymbol{y}_{k}\right), \pi_{k-1}\left(\boldsymbol{p}_{k}\right)\right)+\operatorname{dist}\left(\pi_{k-1}\left(\boldsymbol{p}_{k}\right), \boldsymbol{p}_{k-1}\right) \\
& \leq \delta_{n-k+1}(s) \operatorname{dist}\left(\mathbf{0}, \pi_{k-1}\left(\boldsymbol{p}_{k}\right)\right)+\operatorname{dist}\left(\pi_{k-1}\left(\boldsymbol{p}_{k}\right), \boldsymbol{p}_{k-1}\right) \quad \text { (by } \mathbf{B}_{k} \text { and similarity) } \\
& \leq \delta_{n-k+1}(s) \operatorname{dist}\left(\mathbf{0}, \boldsymbol{p}_{k-1}\right)+\operatorname{dist}\left(\pi_{k-1}\left(\boldsymbol{p}_{k}\right), \boldsymbol{p}_{k-1}\right) \\
& \left.\quad \quad \quad \text { by }\left[\mathbf{0}, \pi_{k-1}\left(\boldsymbol{p}_{k}\right)\right] \subset\left[\mathbf{0}, \boldsymbol{p}_{k-1}\right]\right) \\
& \leq \delta_{n-k+1}(s) \operatorname{dist}\left(\mathbf{0}, \boldsymbol{p}_{k-1}\right)+\operatorname{dist}\left(\pi_{k-1}\left(\boldsymbol{q}_{k}^{+}\right), \boldsymbol{p}_{k-1}\right) \\
& \quad \quad\left(\operatorname{by~}\left[\pi_{k-1}\left(\boldsymbol{p}_{k}\right), \boldsymbol{p}_{k-1}\right] \subset\left[\pi_{k-1}\left(\boldsymbol{q}_{k}^{+}\right), \boldsymbol{p}_{k-1}\right]\right) \\
& \leq \delta_{n-k+1}(s) \operatorname{dist}\left(\mathbf{0}, \boldsymbol{p}_{k-1}\right)+2 \gamma_{n-k+1}(s) \operatorname{dist}\left(\pi_{k-1}\left(\boldsymbol{l}_{k}\right), \pi_{k-1}\left(\boldsymbol{q}_{k}^{0}\right)\right) \quad(\text { by }(2.15)) \\
& \leq\left(\delta_{n-k+1}(s)+2 \gamma_{n-k+1}(s)\right) \operatorname{dist}\left(\mathbf{0}, \boldsymbol{p}_{k-1}\right) . \quad\left(\text { by }\left[\pi_{k-1}\left(\boldsymbol{l}_{k}\right), \pi_{k-1}\left(\boldsymbol{q}_{k}^{0}\right)\right] \subset\left[\mathbf{0}, \boldsymbol{p}_{k-1}\right]\right)
\end{aligned}
$$

Therefore, by (2.2),

$$
\frac{\operatorname{dist}\left(\boldsymbol{y}_{k-1}, P_{k-1}\right)}{\operatorname{dist}\left(\mathbf{0}, P_{k-1}\right)} \leq \delta_{n-k+2}(s)
$$

which is the desired result. This completes the proof of Claim 2.2 .

We have shown that the assumptions $\mathbf{A}_{k-1}, \mathbf{B}_{k-1}$ and $\mathbf{C}_{k-1}$ are satisfied, therefore we can continue the recursion until we arrive at Case $\mathbf{I}$, where we get the desired result (1.2), or else, at worst, arrive at the following scenario. 
2.6. Final remaining possibility: the recursion reaches $k=1$. Suppose that this recursive procedure does not stop before we find $\boldsymbol{p}_{1}, P_{1} \in \boldsymbol{R}^{1}$ satisfying $\mathbf{A}_{1}, \mathbf{B}_{1}$ and $\mathbf{C}_{1}$ and decrease $k$ from 2 to 1 . We now show the desired result can be established in this case. Writing $\boldsymbol{p}_{1}=p_{1}$ to emphasize that we are now dealing with 1-tuples, $\mathbf{A}_{1}$ yields $P_{1}=\left\{p_{1}\right\} \subset R_{1}=\left\{x \in \boldsymbol{R}|| x \mid \leq c_{0}^{n-1} n a^{1}\right\}$, so $2 \operatorname{dist}\left(0, P_{1}\right) \leq$ $\operatorname{diam}\left(P_{1}^{\perp} \cap R_{1}\right)$. Therefore,

$$
\begin{aligned}
\frac{\operatorname{dist}\left(\boldsymbol{y}_{1}, P_{1}\right)}{\operatorname{diam}\left(P_{1}^{\perp} \cap R_{1}\right)} & \leq \frac{\operatorname{dist}\left(\boldsymbol{y}_{1}, P_{1}\right)}{2 \operatorname{dist}\left(0, P_{1}\right)} \\
& \leq \delta_{n}(s) / 2 \quad\left(\text { by } \mathbf{B}_{1}\right) .
\end{aligned}
$$

Since $\mathbf{C}_{1}$ guarantees $\tilde{P}_{1}$ is disjoint from the interior of $S$, we can argue exactly as Case I to show the supporting hyperplane $P$ of $S$ parallel to $\tilde{P}_{1}$ satisfies

$$
\begin{aligned}
\frac{\operatorname{dist}(\boldsymbol{y}, P)}{\operatorname{diam}\left(P^{\perp} \cap S\right)} & \leq n^{3 / 2} \frac{\operatorname{dist}\left(\boldsymbol{y}, \tilde{P}_{1}\right)}{\operatorname{diam}\left(\tilde{P}_{1}^{\perp} \cap R_{n}\right)} \\
& =n^{3 / 2} c_{0}^{n-1} \frac{\operatorname{dist}\left(\boldsymbol{y}_{1}, P_{1}\right)}{\operatorname{diam}\left(P_{1}^{\perp} \cap R_{1}\right)} \\
& \leq n^{3 / 2} c_{0}^{n-1} \delta_{n}(s) / 2 \\
& =n^{3 / 2} c_{0}^{n-1}\left(n-\frac{1}{2}\right) s^{1 / 2^{n-1}}
\end{aligned}
$$

the desired result (1.2).

\section{REFERENCES}

[1] Y. Brenier, Polar factorization and monotone rearrangement of vector-valued functions, Comm. Pure Appl. Math., 44 (1991), pp. 375-417.

[2] L. A. Caffarelli, Some regularity properties of solutions of Monge Ampère equation, Comm. Pure Appl. Math., 44:8-9 (1991), pp. 965-969.

[3] L. A. Caffarelli, The regularity of mapping with a convex potential, J. Amer. Math. Soc., 5 (1992), pp. 99-104.

[4] A. Figalli, Y.-H. Kim, And R. J. MCCann, Hölder continuity and injectivity of optimal maps, to appear in Arch. Rational Mech. Anal.

[5] C. GutiÉRrez, The Monge-Ampère Equation, Birkhauser, 2001.

[6] F. John, Extremum problems with inequalities as subsidiary conditions, Studies and Essays Presented to R. Courant on his 60th Birthday, January 8, 1948, pp. 187-204. Interscience Publishers, Inc., New York, N. Y., 1948.

[7] N. Trudinger and X.-J. Wang, On the second boundary value problem for Monge-Ampère type equations and optimal transportation, Ann. Sc. Norm. Super. Pisa Cl. Sci. (5), 8:1 (2009), pp. 143-174.

[8] C. Villani, Optimal Transport, Old and New, Grundlehren des mathematischen Wissenschaften [Fundamental Principles of Mathematical Sciences], Vol. 338, Springer-Verlag, Berlin-New York, 2009. 
